\author{
Military Technical College \\ Kobry Elkobbah, \\ Cairo, Egypt
}

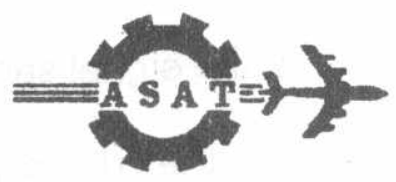

$8^{\text {th }}$ International Conference on Aerospace Sciences \&

Aviation Technology

\title{
Modifications to Pisarenko Harmonic Retrieval Method to Cope with Rough Noise Channels Conditions
}

\author{
Nabil A. Amin*
}

Abstract: Pisarenko method is an important technique to estimate the sinusoidal signal frequencies in white noise. In this paper, we show that the assumption of fair white noise and its corresponding equal values diagonal covariance matrix can be perturbed in some noise environments. Therefore, the simple criterion form of Pisarenko is not suitable and other modified algorithms are to be proposed to alleviate this problem. These algorithms and their results are shown.

\section{Introduction:}

Pisarenko method is one of the important techniques to estimate the sinusoidal signals frequencies in white noise [1], [2]. A problem of strong interest in the digital signal processing applications. The method is based on finding the optimal weighting vector corresponding to the minimum eigenvalue of the signal sampled covariance matrix.

The interested signal sample is represented by:

$$
x(n)=\sum_{j=1}^{m} C_{j} \sin \left(2 \pi f_{j} \cdot n+\phi_{j}\right)+z(n)
$$

$C_{j}, f_{j}, \phi_{j}$ are the amplitude, frequency (to be estimated) and phase of the $j^{\text {th }}$ sinusoidal signal and $z(n)$, the additive white gaussian noise, zero mean and variance $\sigma_{n}^{2}$ (for this particular signal, SNR $=10 \log \frac{C_{j}^{2}}{2 \sigma_{n}^{2}}$ ) For the signal vector $X_{i}$

$$
\mathrm{X}_{\mathrm{i}}^{\mathrm{T}}=[\mathrm{x}(\mathrm{i}), \mathrm{x}(\mathrm{i}-1), \ldots \ldots \ldots \ldots \ldots . . . \mathrm{x}(\mathrm{i}-\mathrm{N}+1)]
$$

(T: vector transpose)

The covariance matrix is given by:

$$
R_{x x}=\frac{1}{(L-N+1)} \sum_{i=N}^{L} X_{i} X_{i}^{T}
$$

* Lecturer, Dpt. of Electronics and Communications, Faculty of Engineering, Cairo University, Giza, Egypt 
where $L$ is the number of the signal samples and $N$ is the dimension of the signal vector

Pisarenko is mainly optimizing the criterion

$$
\min _{W} \rho=\frac{W^{T} R_{x x} W}{W^{T} W}
$$

The minimum of $\rho$ is known as the minimum eigenvalue of $R_{x x}$ and $W$ is the corresponding eigenvector. $W$ is used as the weighting vector in the spectrum estimator of the signals in (1). This covariance matrix $\left(R_{x \alpha}\right)$ can be considered as the sum of the sinusoidal signals covariance matrix plus the noise covariance matrix as:

$$
\mathrm{R}_{\mathrm{xx}}=\mathrm{R}_{\mathrm{s}}+\mathrm{R}_{\mathrm{n}}
$$

This method is based on the assumption that the noise is white, i.e. its covariance matrix is given by:

$$
R_{n}=\sigma_{n}^{2} I
$$

(I: identity matrix)

So, the optimal vector to (3) is given by:

$$
\mathrm{W}=\frac{1}{\mathrm{~L}} \cdot \sum_{\mathrm{i}=1}^{\mathrm{L}} \mathrm{V}_{\mathrm{i}}
$$

i.e. the sum of the noise eignevectors whose eignevalues are the same and equal to $\sigma_{\mathrm{n}}^{2}\left(\sigma_{\mathrm{n}}^{2}\right.$ is the least value of $\left.(3)\right)$, where

$$
\begin{aligned}
& R_{s} V_{i}=0 \\
& R_{n} V_{i}=\sigma_{n}^{2} V_{i}
\end{aligned}
$$

(Thus, the weighting polynomial $W(\omega)$ based on $W$ has zeros at the sinuscidal signals frequencies). $R_{x x}$ has $N$ - dimension where $L=N-2 m$. We found that this condition on the additive noise may not completely fulfilled in scrne cases, as $R_{n}$ has small perturbed matrix from the assumed identity rnatrix or:

$$
R_{n}=\sigma_{n}^{2} 1+\Delta R
$$

; as $\Delta \mathrm{R}$ is perturbation matrix.

Therefore, the corresponding noise eigenvectors and their eigenvalues are as well perturbed and not equal as changed from the assumed case in (B). Actually, while solving the criterion in (3), the algorithm is terminated to single noise eigenvector corresponding to the lowest eigenvalue or the most perturbed one. Again, this situation is different from the assumed case to obtain in (6). The obtained results are usually showing spurious noise to smear the wanted results. This situation is precisely met while we perform some experiments using linear congruential equation that is custom to use in signal processing to generate the white noise, this subroutine can be found as in [3]. The noise correlation lags are found as:

$$
r_{z}(1)=0.952, \quad 0.0627,-0.0792,-0.1008,-0.0337,-0.0315-0.0791 \text {, }
$$
0.0736

$$
\text { (1) }=0,1,2, \quad, 7, \ldots
$$


while the ideal noise is assumed to have correlations lags close to $(1,0, \ldots 0)$. We believe similar situations can be found in some practical noisy channels. It is the purpose of this paper to suggest the necessary modifications to (3) to avoid such fautly results in these noise circumstances.

The following section contains the derived algorithms. The simulation results to show the algorithms results and improvements are presented in section (3). Finally, the conlcusion is given.

\section{2- The proposed algorithms:}

To correct the pitfall of the criterion in (3) in the explained noise conditions, we modified the problem in (3) from looking for vector $W$ corresponding to the minimum eigenvalue to find $W$ that gives $\rho$ equal to $\sigma_{n}^{2}$, i.e. find $W$ s.t.

$$
\rho=\frac{W^{\mathrm{T}} \mathrm{R}_{\mathrm{xx}} \mathrm{W}}{\mathrm{W}^{\mathrm{T}} \mathrm{W}}=\sigma_{\mathrm{n}}^{2}
$$

Therefore, the algorithms require prior knowledge of the noise variance. This noise variance can be estimated in a simple way as solving the set of equations corresponding to overdetermined autoregressive model, i.e.

$$
r_{x}(q)+\sum_{i=1}^{N} C_{i} r_{x}(q-i)=0 \quad, N+1 \leq q \leq 2 N
$$

and based on these coefficients, the noise variance is estimated as:

$$
\sigma_{n}^{2}=\left[\sum_{i=1}^{N} C_{i}\left[r_{x}(i)+\sum_{i=1}^{N} C_{i} r_{x}(i-j)\right]\right] / \sum_{i=1}^{N} C_{i}^{2}
$$

where the correlation lag is given by:

$$
r_{x}(m)=\frac{1}{L-m} \sum_{i=1}^{L-m} x(i) x(i+m)
$$

Accordingly, we suggest the following algorithms.

\section{Algorithm (1):}

The criterion in (3) is modified to the following

$$
\min \frac{W^{T}\left(R_{x x}-\sigma_{n}^{2} I\right)\left(R_{x x}-\sigma_{n}^{2} I\right) W}{W^{T} W}
$$

The matrix $B=\left(R_{x x}-\sigma_{n}^{2} I\right)\left(R_{x x}-\sigma_{n}^{2} I\right)$ has the same eigenvectors as $R_{x x}$. However, the minimum of (15) attained is zero to give the same vector of (3) corresponding to $\rho=\sigma_{\mathrm{n}}^{2}$. This is the solution to cited problem in (11) and the obtained vector belongs to the noise subspace without being one of the noise eigenvectors. The following numerical algorithm is used to solve (15). It is based on the conjugate gradient vector, it is better search direction vector than gradient vector (this algorithm is used in similar problems [4], [5]) defined as:

$$
P_{n}=G_{n}+\alpha_{n} P_{n-1}
$$

where $G_{n}$ is the gradient vector of (15) and is given by: 


$$
\begin{aligned}
& G_{n}=\frac{-2}{W_{n}^{T} W_{n}}\left[B-\rho_{n} I\right] W_{n} \\
& \alpha_{n}=-\frac{\left[G_{n}-G_{n-1}\right]^{T} G_{n}}{\left[G_{n}-G_{n-1}\right]^{T} P_{n-1}}
\end{aligned}
$$

$\rho_{n}=$ value of (15) with $W_{n}$, and as the successive search vectors are conjugate orthogonal or restricted by:

$$
\left[\mathrm{G}_{\mathrm{n}}-\mathrm{G}_{\mathrm{n}-1}\right]^{\mathrm{T}} \mathrm{P}_{\mathrm{n}}=0
$$

To update the vector $\mathrm{W}_{n}$;

$$
\mathrm{V}_{\mathrm{n}+1}=\mathrm{W}_{\mathrm{n}}+\mathrm{t}_{\mathrm{n}} \mathrm{P}_{\mathrm{n}}
$$

where $t_{n}$ is the step size at which the criterion (15) is minimum,

and $\mathrm{W}_{\mathrm{n}+1}=\frac{\mathrm{V}_{\mathrm{n}+1}}{\left\|\mathrm{~V}_{\mathrm{n}+1}\right\|} ;\left\|\mathrm{V}_{\mathrm{n}+1}\right\|=\sqrt{\mathrm{V}_{\mathrm{n}+1}^{\mathrm{T}} \cdot \mathrm{V}_{\mathrm{n}+1}}$

as the optimum of $W_{n}$ is reached when $\left\|G_{n}\right\| \approx \in\left(\epsilon=10^{-8}\right)$

The objective function value in (15) is scale invariant w.r.t. W. The convergence characteristics to the optimal point is better with this vector normalization.

\section{Algorthim (III):}

The solution to the problem arised in (11) can be obtained as power inversion technique solution that:

and

$$
\mathrm{V}_{\mathrm{k}+1}=\left(\mathrm{R}_{\mathrm{xx}}-\sigma_{\mathrm{n}}^{2} \mathrm{I}\right)^{-1} \mathrm{~W}_{\mathrm{k}}
$$

and

$$
\mathrm{W}_{\mathrm{k}+1}=\frac{\mathrm{V}_{\mathrm{k}+1}}{\left\|\mathrm{~V}_{\mathrm{k}+1}\right\|}
$$

Through this iterative procedure, the emphasized components results in $W_{k+1}$ will be the solution of (11). The normalization of the weighting vector is found through experiments to enhance the results further. The initial vector can be any nonzero vector. It is to be noticed that it is very simple to obtain the inverse matrix in (21) iteratively as:

$$
R_{L}^{-1}=\left(R_{x x}-\sigma_{n}^{2} I\right)^{-1}=L\left(\sum_{i=1}^{L} X_{i} X_{i}^{T}-L \sigma_{n}^{2} I\right)^{-1}
$$

and for the first sample vector, we have

$$
\begin{aligned}
& R_{1}^{-1}=\left(X_{1} X_{1}^{T}-L \sigma_{n}^{2} I\right)^{-1}=\frac{-1}{L \sigma_{n}^{2}}\left(I+\frac{X_{1} X_{1}^{T}}{L \sigma_{n}^{2}-X_{1}^{T} X_{1}}\right) \\
& ; R_{2}^{-1}=\left(X_{2} X_{2}^{T}+X_{1} X_{1}^{T}-L \sigma_{n}^{2} I\right)^{-1}=R_{1}^{-1}-\frac{R_{1}^{-1} X_{2} X_{2}^{T} R_{1}^{-1}}{1+X_{2}^{T} R_{1}^{-1} X_{2}}
\end{aligned}
$$

a'nd so on until $\mathrm{R}_{\mathrm{L}}^{-1}$. 
Algorithm (II):

An adaptive loop is derived to converge to the solution of (11) as:

$$
\begin{aligned}
& \mathrm{V}_{\mathrm{k}+1}=\mathrm{W}_{\mathrm{k}}-\mu\left[\mathrm{R}_{\mathrm{xx}}-\sigma_{\mathrm{n}}^{2} \mathrm{I}\right] \mathrm{W}_{\mathrm{k}} \\
& ; \mathrm{W}_{\mathrm{k}+1}=\frac{\mathrm{V}_{\mathrm{k}+1}}{\left\|\mathrm{~V}_{\mathrm{k}+1}\right\|} \quad, \quad \mu=\frac{1}{\text { trace of } \mathrm{R}_{\mathrm{xx}}}
\end{aligned}
$$

In this adaptive mode, the eigenvectors of $R_{x x}$ with eigenvalues larger than $\sigma_{\mathrm{n}}^{2}$ will have less than one eigenvalue in the loop equation to be attenuated. On the other hand, the noise eigenvectors will have eigenvalue in the loop equation larger than one to stay as the converged vector approach the solution of (11). The performance of these alogrithms in the noise conditions explained in (10) are shown in the next section.

\section{3- Simulation results} given by:

The estimated power spectral density (PSD) of the signals in (1), is

$$
P(\omega)=\frac{1}{\left[\sum_{i=1}^{N} w_{i} e^{-j(i-1) \omega}\right]^{2}} \quad,-\pi \leq w \leq \pi
$$

$W_{i}$ is the $i^{\text {th }}$ component of the optimal weighting vector $W . A$ simulated signal is used with two sinusoidal signals, $f_{1}=0.27, f_{2}=0.34$ (the frequencies are normalized w.r.t. the sampling frequency) with $\mathrm{SNR}=5,10 \mathrm{~dB}$ respectively w.r.t. $\sigma_{n}^{2}=1$. To represent the noise " $z(n) "$, linear congruential random generator is used as:

$$
\text { and } \begin{aligned}
\mathrm{I}_{\mathrm{n}} & =\left(\mathrm{a} \mathrm{I}_{\mathrm{n}}+1\right) \bmod (\mathrm{m}) \\
\mathrm{u}_{\mathrm{n}} & =\frac{\mathrm{I}_{\mathrm{n}}}{\mathrm{m}}-0.5
\end{aligned}
$$

$u_{n}$ has uniform distribution over the range; $-0.5 \leq u_{n} \leq 0.5$. In this particular example, $a=493$ and $m=2^{20}$. To obtain $z(n)$, each 12 successive samples of $u_{n}$ are sumed. In Fig. (1), Algorithm (I) is applied and the resulted spectrum is shown for $\mathbf{N}=20$. It takes 9 iterations to reach the optimal value as the results shows two sharp peaks at the correct frequencies. For the same signals, when applied to pisarenko method criterion in (3), the unsatisfactory performance results are shown in Fig. (4). In Fig. (2), the results are based on Alg. (II), two sharp peaks are again identified at the sinusoidals frequencies, however there is uncorrect false peak at $f=0.5$, perhaps due to the noise and the nonlinearity of the algorithm. Fig. (3-a) shows good results for the adaptive loop Alg. (III) to estimate the sinusoidal frequencies by identifying their corresponding sharp peaks. The convergence characteristics of this adaptive algorithm is shown in Fig. (3-b) as $\rho$ versus $n$ (iteration number) to show descent and fast behavior to reach the steady state as designed. 


\section{4- Conclusion:}

The criterion of Pisarenko harmonic retrieval method is modified to accommodate more noise channels conditions. The new criterion is to obtain the weighting vector corresponding to $\rho=\sigma_{n}^{2}$ (noise variance). Three different algorithms are introduced to find this weighting vector. Simulation results to show the advarice of these algorithms are presented. Algorithm (II) \& (III) are simple to be implemented than algorithm (I). However, the results are better for algorithm (I) \& (III) with respect to the results resolution and immunity to noise false peak.

\section{References:}

1. S.H. Kay and L. Marple, "Spectrum analysis: A modern perspective", Proc. IEEE, vol. 69, pp. 1380-1418, Nov. 1981.

2. B.D. Rao and K.S. Arun, "Model Based processing of Signals: A state space approach". Proc. IEEE, vol. 80, pp. 283-309, Feb 1992.

3. B. Widrow and S. Stearns, Adaptive Signal processing, Englewood Cliffs, NJ.: Prentice - Hall, 1985.

4. H. Chen et al, "Adaptive spectral estimation by the conjugate gradient method", IEEE Trans. Acoust. Speech, Signal processing, vol. ASSP-34, pp. 272-284, Apr. 1986.

5. X. Yang et al, "A survey of conjugate gradient algorithms for solution of Extreme Eigen. Problems of a symmetric Matrix" IEEE Trans. Acoust, Speech, Sigrıal Processing vol. ASSP-37 pp. 1550-1556, Oct. 1989.

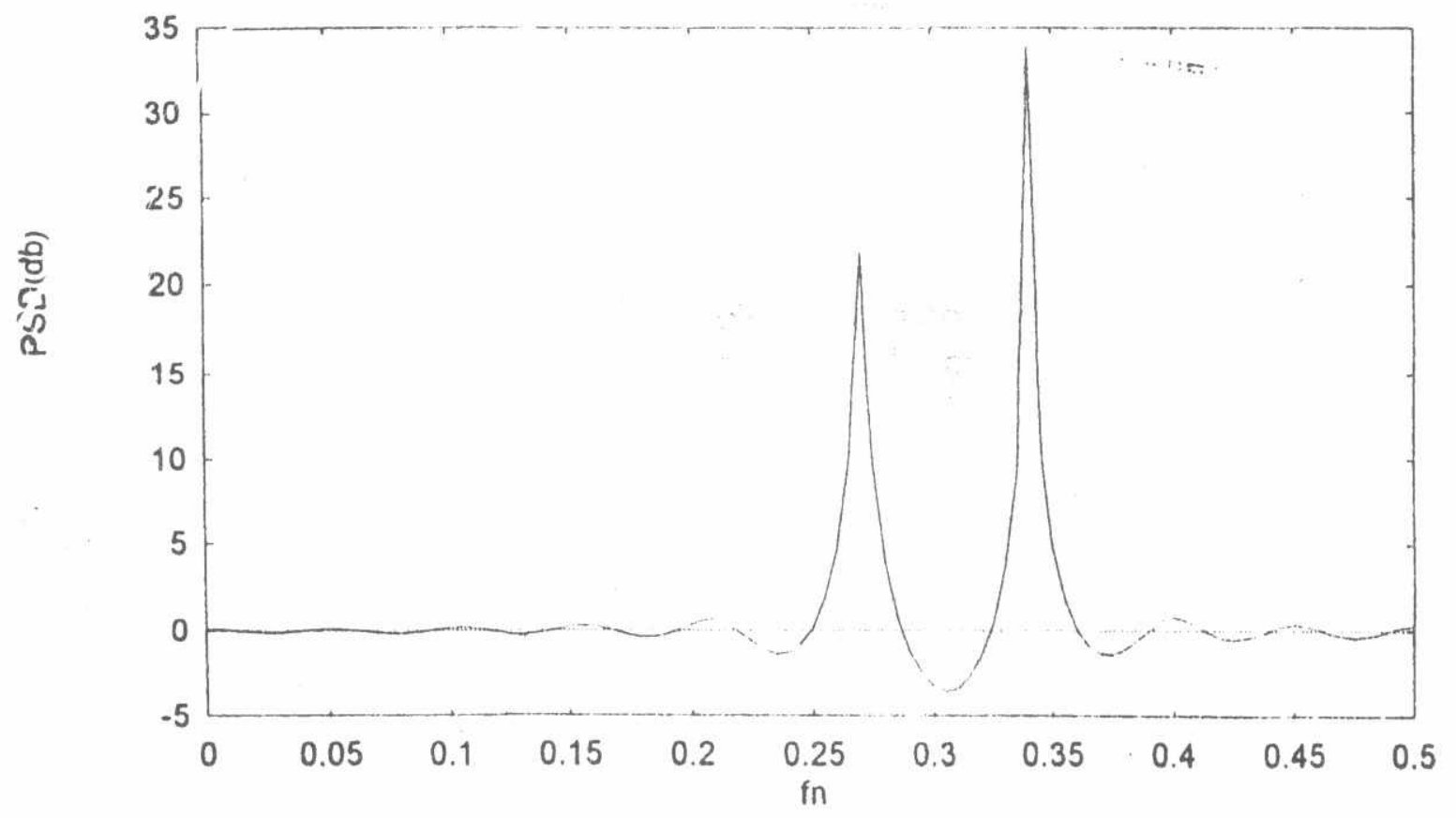

Fig. (1) Alg. I, $N=20$ 

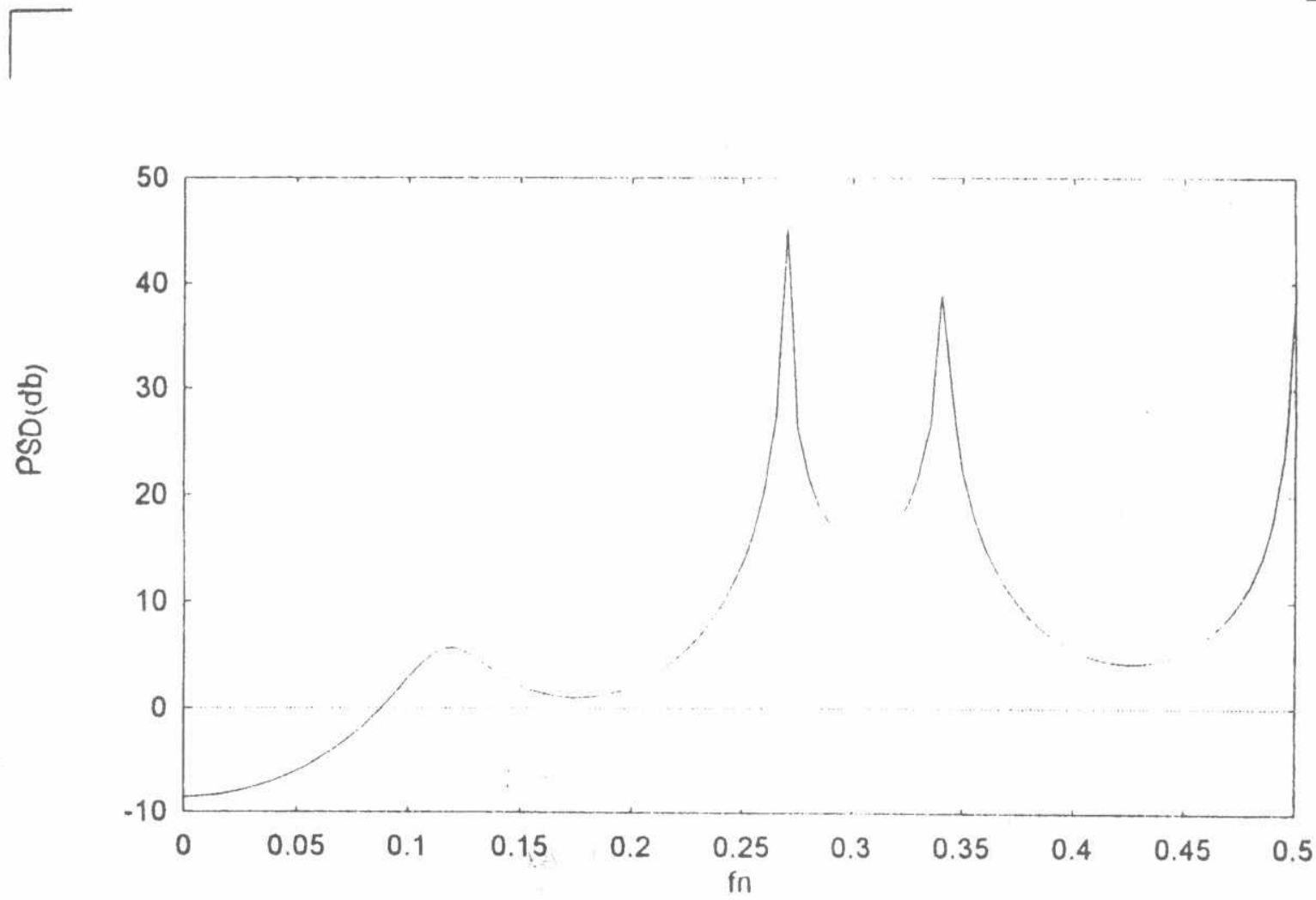

Fig. (2) Alg. II, $N=8$

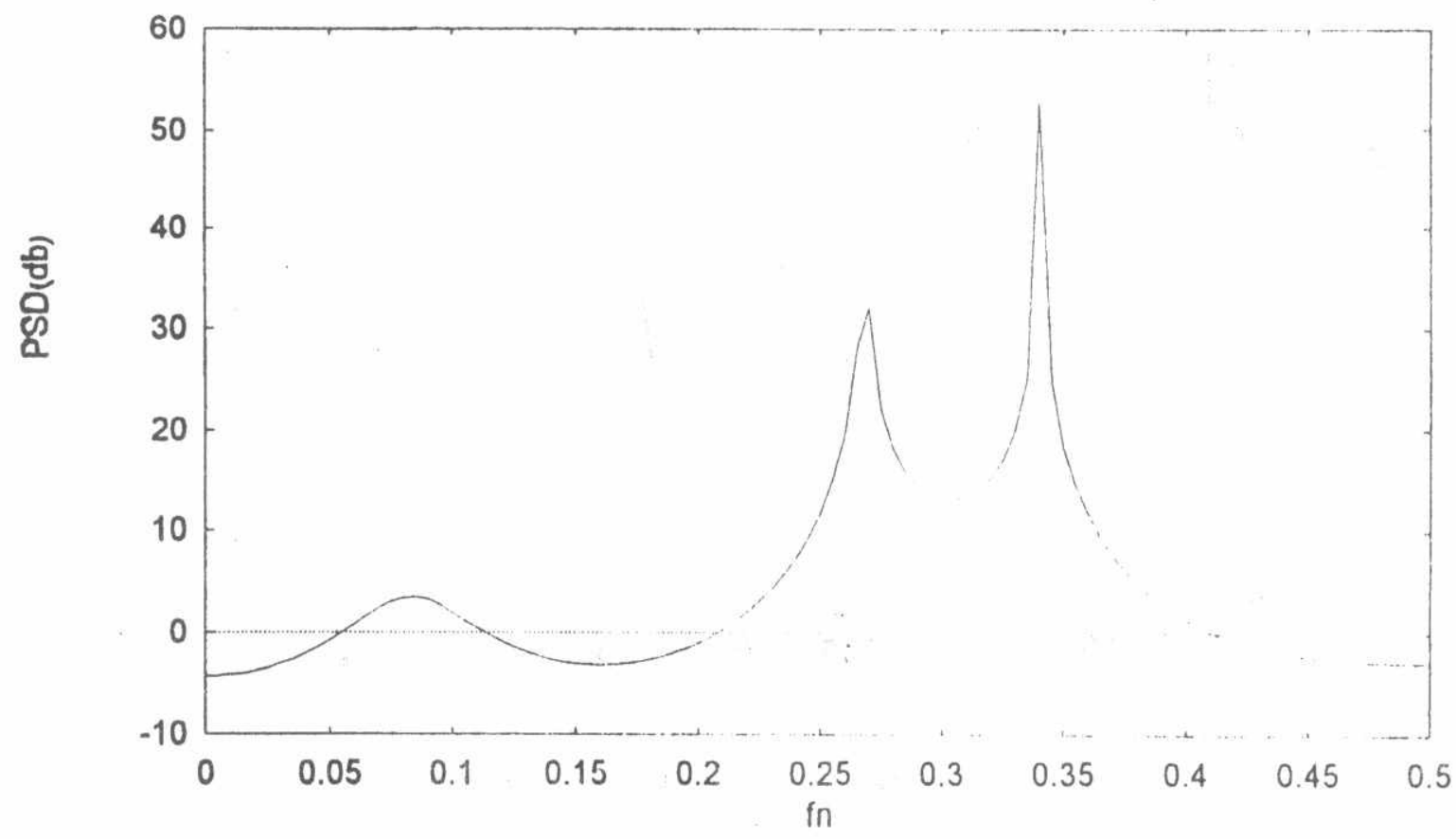

Fig. (3-a) Alg. III, $N=8$ 


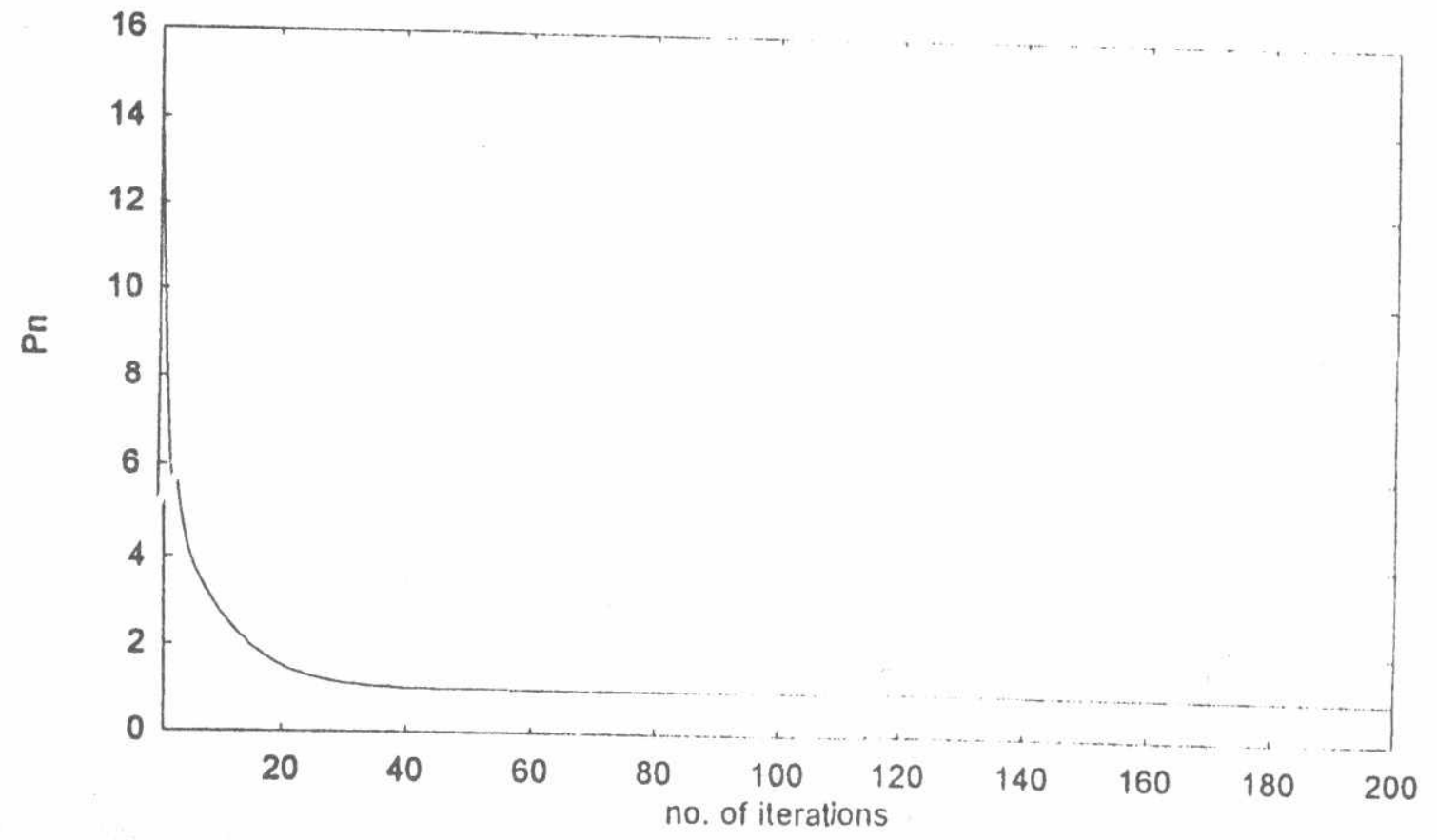

Fig. (3-b) AIg. III, $N=8$

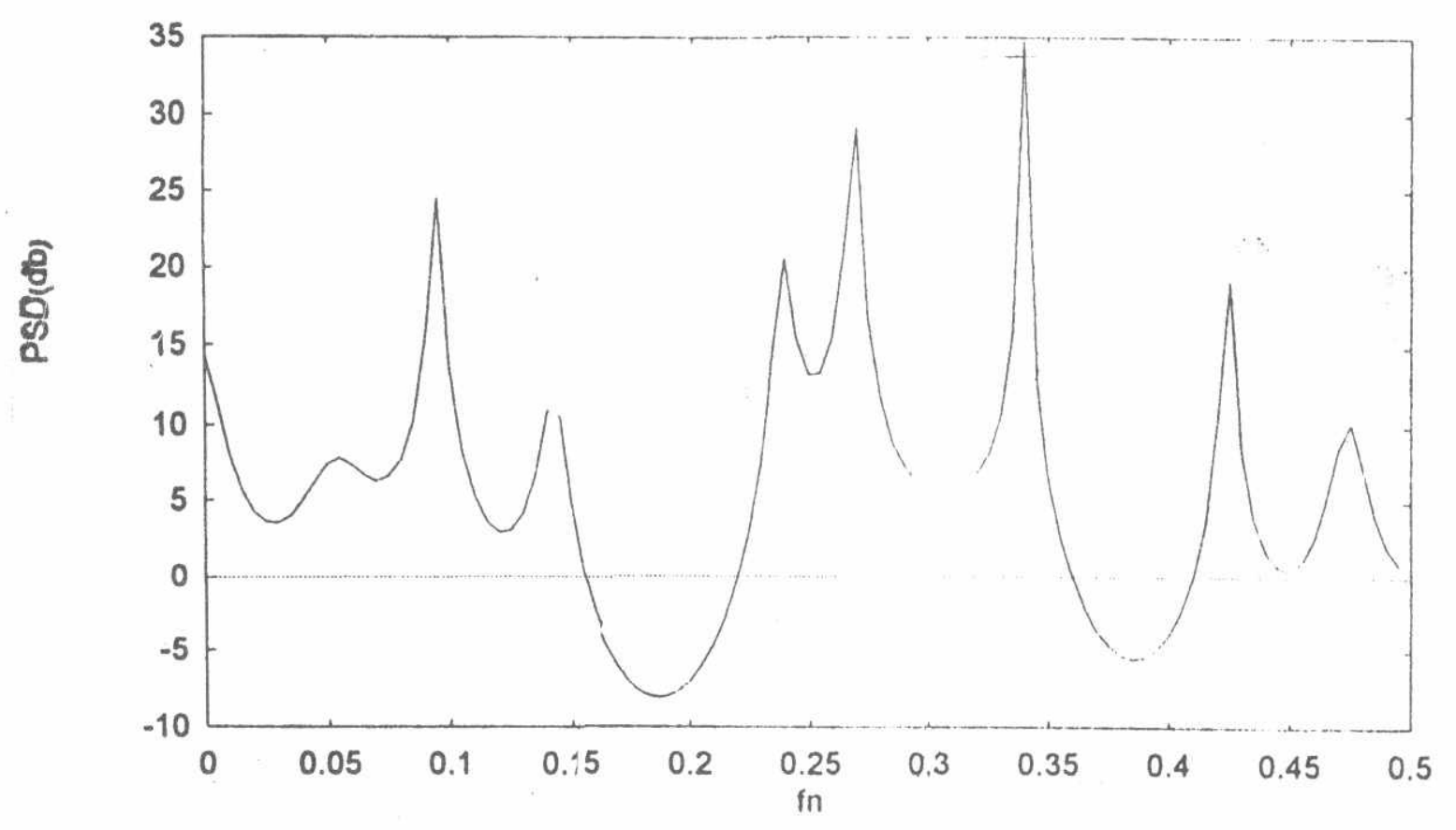

Fig. (4) Alg. I with $\left(\rho_{m}\right), N=20$ 